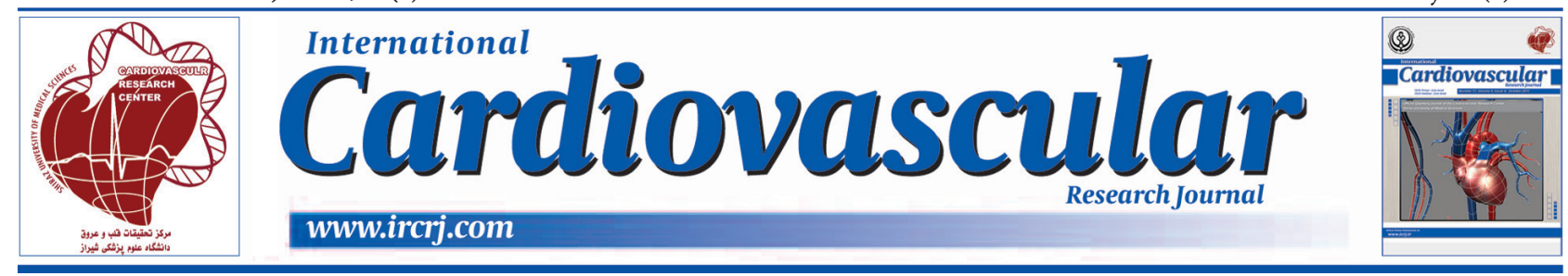

\title{
Baseline Depressive Symptoms Predict Subsequent Heart Disease; A 20-Year Cohort
}

\author{
Maryam Moghani Lankarani ${ }^{1,2}$ and Shervin Assari ${ }^{1,2, *}$
}

${ }^{1}$ Department of Psychiatry, University of Michigan, School of Medicine, Ann Arbor, MI

${ }^{2}$ Center for Research on Ethnicity, Culture and Health (CRECH), University of Michigan, School of Public Health, Ann Arbor, MI

\begin{tabular}{l}
\hline A R T I C L E I N F O \\
\hline Article Type: \\
Research Article \\
\hline
\end{tabular}

Article History:

Received: 29 Nov 2014

Revised: 15 Jun 2015

Accepted: 23 Jun 2015

\section{Keywords:}

Depression

Heart Disease

Smoking

Exercise

\begin{abstract}
A B S T R A C T
Background: Depression is common among patients with heart disease. Depression is also associated with worse outcomes among patients with heart disease. Fewer studies have shown whether or not baseline depressive symptoms predict subsequent heart disease in general population.

Objectives: This study aimed to investigate whether depressive symptoms at baseline predict risk of developing heart disease during the next 20 years in the United States.

Patients and Methods: The data were extracted from the Health and Retirement Study (HRS), 1992 - 2012. The study was conducted on 8,375 individuals who were older than 50 years at entry, did not have heart disease at baseline, and had data on heart disease over the next 20 years. High depressive symptoms (modified Center for Epidemiologic Studies Depression Scale [CES-D]) were considered as the independent variable. Self-reported data on physician diagnosis of heart disease were measured on a biannual basis. Baseline demographic data (i.e., age and gender), socioeconomic status (i.e., race, marital status, and education level), health behaviors (i.e., drinking, smoking, and exercise), and body mass index were controlled. Cox proportional hazard model was used for data analysis. Results: Cox proportional hazard model revealed a link between high depressive symptoms at baseline and time to developing heart disease (Hazard ratio $=1.439,95 \% \mathrm{CI}=1.253$ 1.652), suggesting that individuals with high depressive symptoms at baseline developed heart disease sooner than others. The association between baseline depressive symptoms and risk of heart disease was significant after controlling for all the covariates.

Conclusions: Individuals with depressive symptomatology are at higher risk of development of heart disease over time. Thus, individuals with depressed mood may need more rigorous evaluation for heart disease.
\end{abstract}

Implication for health policy/practice/research/medical education:

As depressed mood contributes to development of heart disease, individuals with depressed mood may need more rigorous evaluation of heart disease.

\section{Background}

The association between depression and prognosis of cardiovascular disease is well established among patients who have already developed the disease (1). Depression and high depressive symptoms are both associated with an increased risk of cardiovascular morbidity and mortality among patients with Coronary Artery Disease (CAD) (27). Reviews and meta-analyses have shown that the effect of depression on outcomes of CAD is relatively consistent

*Corresponding author: Shervin Assari, Research Faculty, Department of Psychiatry, School of Medicine, University of Michigan, Ann Arbor, MI. Tel: + 1-7342320445, Fax: + 1-7346158739,

E-mail: assari@med.umich.edu
(8-11). Despite availability of strong evidence on the link between depression and outcomes among patients with heart disease, the effect of depression on cardiovascular disease in general population is still not clear (12). Some (13-15), but not all $(14,16)$ studies have shown positive results. For instance, a prospective cohort of 136,293 community-dwelling postmenopausal women revealed that new antidepressant use was not significantly associated with increased incidence of CAD (16). Another study also failed to find an effect of antidepressant use on the risk of heart failure in patients with CAD (14). Barrick argued that it is premature to describe depression as a cause of 
heart disease (17). Appels also argued that more research is needed before concluding about a causal link between depression and heart disease (18).

\section{Objectives}

The present study aims to determine whether individuals with high levels of depressive symptoms at baseline are at a higher risk of developing heart disease during the next 20 years in the United States.

\section{Patients and Methods}

This study used Health and Retirement Study (HRS) data to assess the effect of high depressive symptoms at baseline on development of heart disease in the United States. The HRS is a longitudinal cohort that has enrolled a representative sample of Americans over the age of 50 . Since its launch in 1992, the study has interviewed the participants for 20 years every two years. The HRS explores the health transitions that individuals undergo toward the end of their work lives and in the following years. The HRS has collected information about socioeconomic status, physical health, functioning, mental health, and healthcare utilization. University of Michigan Institutional Review Board approved the study. Study design and sampling have been described in details elsewhere $(19,20)$.

\subsection{Participants}

The participants of the HRS cohort included individuals who were born between 1931 and 1941 and were 51 to 61 years old. The first interview happened in 1992. Only 8,375 individuals who did not have heart disease at baseline and had data on all waves during the next 20 years were enrolled into the current study.

\subsection{Interviews}

The data were collected using face-to-face interviews. The interviews were conducted in the participants' houses, and the participants received compensation for taking part in the study.

\subsection{Measures}

\subsubsection{Demographic Data}

The data on race, ethnicity, age, gender, education level, and marital status were used. The respondents' race was asked if they considered themselves White or Caucasian, Black or African-American, American-Indian, Asian, or something else. Besides, ethnicity included Hispanic or Latino.

\subsubsection{Depressive Symptomatology}

A modified nine-item version of the Center for Epidemiologic Studies Depression Scale (CES-D) was used to measure depressive symptoms (21). The participants reported whether they had felt (a) depressed, (b) everything was an effort, (c) sleep was restless, (d) happy, (e) lonely, (f) that they enjoyed life, (g) sad, (h) that they could not get going, and (i) that they had a lot of energy during the past week. Factor analysis studies have identified the following three subscales for this measure: somatic (items 2, 3, 8, and 9), lack of positive affect (items 4 and 6), and dysphoria (items 1, 5, and 7) (22). In this scale, higher scores reflected negative affect, and scores 4 and above represented high depressive symptoms. The measure displayed acceptable internal consistency $(\mathrm{a}=0.80-0.83)(23)$.

\subsubsection{Heart Disease}

The participants reported if a doctor had ever told them that they had any heart conditions, including heart attacks, angina, congestive heart failure, or coronary heart disease. If the respondents reported "yes" in the previous wave of data and "no" in the current wave, their response was coded as "no" (24).

\subsubsection{Smoking}

Two items were used to measure smoking behavior at baseline. In the first item, ever smoking and in the second item, current smoking was asked. Using both items, the individuals were categorized into never smokers, previous smokers, and current smokers.

\subsubsection{Exercise}

Three items were used to measure physical activity and exercise. Cronbach's alpha of the measure was 0.792 .

\subsection{Statistical Note}

Due to the complex sample design, Stata 13.0 (Stata Corp., College Station, TX) was used for data analysis. Taylor series linearization was used for estimation of standard errors. Thus, stratified and clustered nature of the data was considered for data analysis. P values less than 0.05 were considered to be statistically significant. The data were downloaded from the HRS website, Institute for Social Research (ISR), University of Michigan (http://hrsonline. isr.umich.edu/).

Cox proportional hazard model was used to determine whether the association between high depressive symptoms and time to developing heart disease is significant after controlling the effects of baseline demographic data, socioeconomic status, health behaviors, and Body Mass Index (BMI). Covariates included age, gender, race, ethnicity, education level, marital status, smoking, physical activity, drinking, and BMI. Hazard Ratios (HR) with 95\% CI were reported. HRs greater than 1 suggests higher risk of developing heart disease at each time point.

The possible multi-collinearity between the covariates was also tested. With considering correlation coefficients of larger than 0.4 as indicator of collinearity, no sign of multicollinearity between the predictors was found. Additionally, Kaplan-Meier curves were used to depict how risk of developing heart disease over time varied based on race, gender, smoking, and depressive symptoms.

\section{Results}

In this study, most of the participants were female (54\%) and White and non-Hispanic (95\%). Besides, $42 \%$ were never smokers, 38\% were past smokers, and 19\% were current smokers. In addition, $68 \%$ reported drinking alcohol. About $8 \%$ had high depressive symptoms and $42 \%$ developed new CAD during the 20-year follow-up (Table 1).

Kaplan-Meier curves showed that the risk of developing heart disease was higher among males compared to females 


\begin{tabular}{|c|c|c|c|c|}
\hline Socioeconomics & $\%$ & Linearize d Std. & \multicolumn{2}{|c|}{ 95\% Confidence Interval, $(n=8,375)$} \\
\hline \multicolumn{5}{|l|}{ Gender } \\
\hline Male & 45.49 & 0.008 & 43.96 & 47.01 \\
\hline Female & 54.51 & 0.008 & 52.99 & 56.04 \\
\hline \multicolumn{5}{|l|}{ Race } \\
\hline White & 95.13 & 0.005 & 94.14 & 96.12 \\
\hline Black & 4.87 & 0.005 & 3.88 & 5.86 \\
\hline \multicolumn{5}{|l|}{ Ethnicity } \\
\hline Non-Hispanic & 95.42 & 0.008 & 93.81 & 97.03 \\
\hline Hispanic & 4.58 & 0.008 & 2.97 & 6.19 \\
\hline \multicolumn{5}{|l|}{ Behaviors } \\
\hline \multicolumn{5}{|l|}{ Smoking } \\
\hline Never & 42.42 & 0.011 & 40.15 & 44.69 \\
\hline Current & 38.27 & 0.01 & 36.21 & 40.33 \\
\hline Drinking & 19.31 & 0.009 & 17.44 & 21.17 \\
\hline No & 32.41 & 0.014 & 29.55 & 35.28 \\
\hline Yes & 67.59 & 0.014 & 64.27 & 70.45 \\
\hline \multicolumn{5}{|l|}{ Predictor } \\
\hline Depressive symptoms & 92.41 & 0.005 & 91.43 & 93.4 \\
\hline \multicolumn{5}{|l|}{ Low } \\
\hline High & 7.59 & 0.005 & 6.6 & 8.57 \\
\hline \multicolumn{5}{|l|}{ Outcome } \\
\hline \multicolumn{5}{|l|}{ New heart disease } \\
\hline No & 57.8 & 0.012 & 55.38 & 60.23 \\
\hline Yes & 42.2 & 0.012 & 39.77 & 44.62 \\
\hline
\end{tabular}

(Figure 1), among African-Americans compared to Whites (Figure 2), among smokers compared to non-smokers (Figure 3), and among the individuals with high depressive symptoms (Figure 4). Moreover, Cox proportional hazard model confirmed that the association between high level of depressive symptoms and time to development of heart disease was statistically significant $(\mathrm{HR}=1.439,95 \% \mathrm{CI}$ $=1.253-1.652$ ) after controlling for demographic data, socioeconomic status, health behaviors, and BMI (Table 2).

\section{Discussion}

The results of the current longitudinal study showed that high

Figure 1. Kaplan-Meier Graph Showing Hazard of Developing Heart Disease Over 20 Years Based on Gender

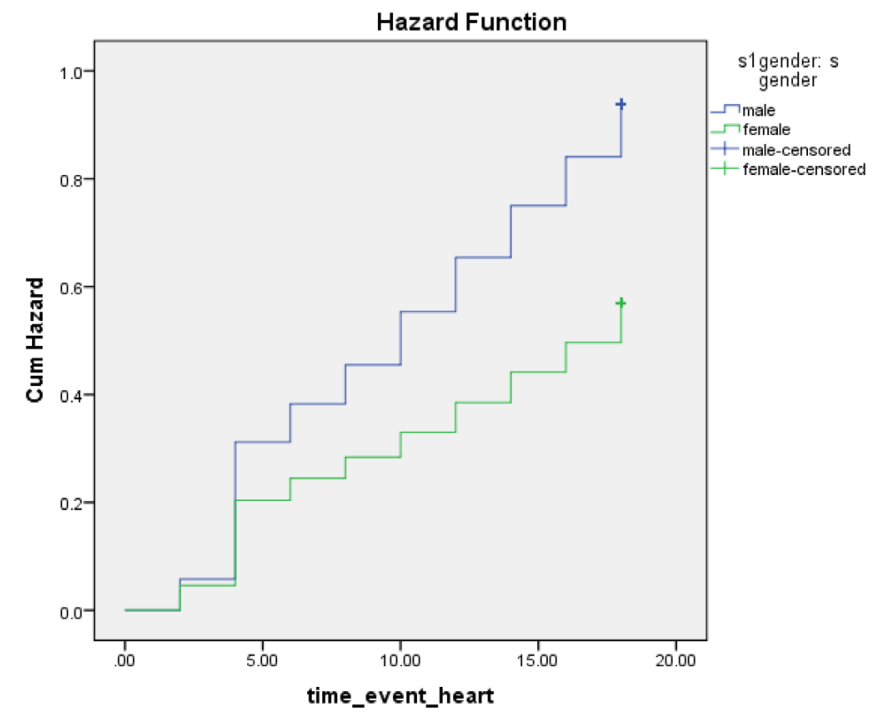




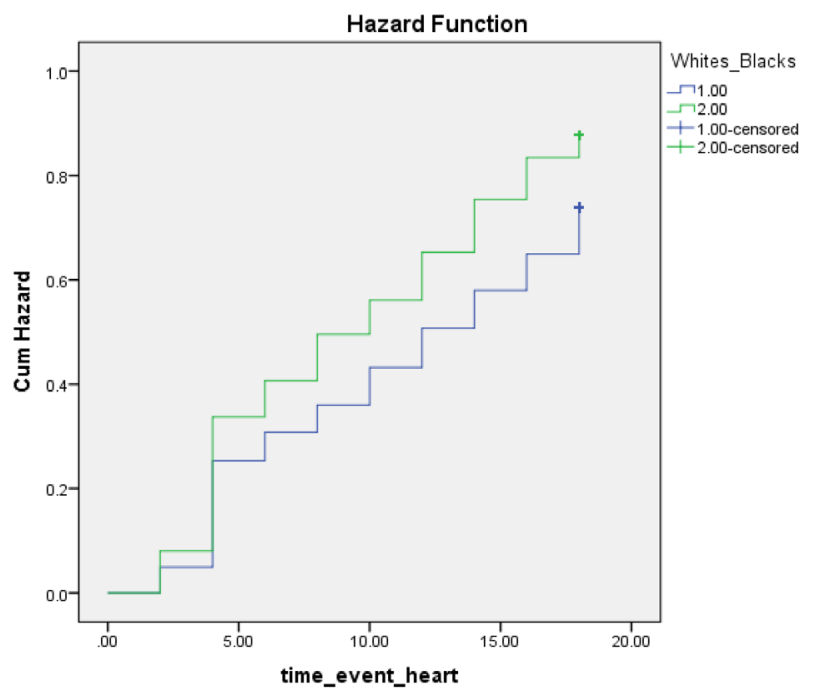

Whites $=$ Blue, Blacks $=$ Green

Figure 3. Kaplan-Meier Graph Showing Hazard of Developing Heart Disease Over 20 Years Based on Smoking Status at Baseline

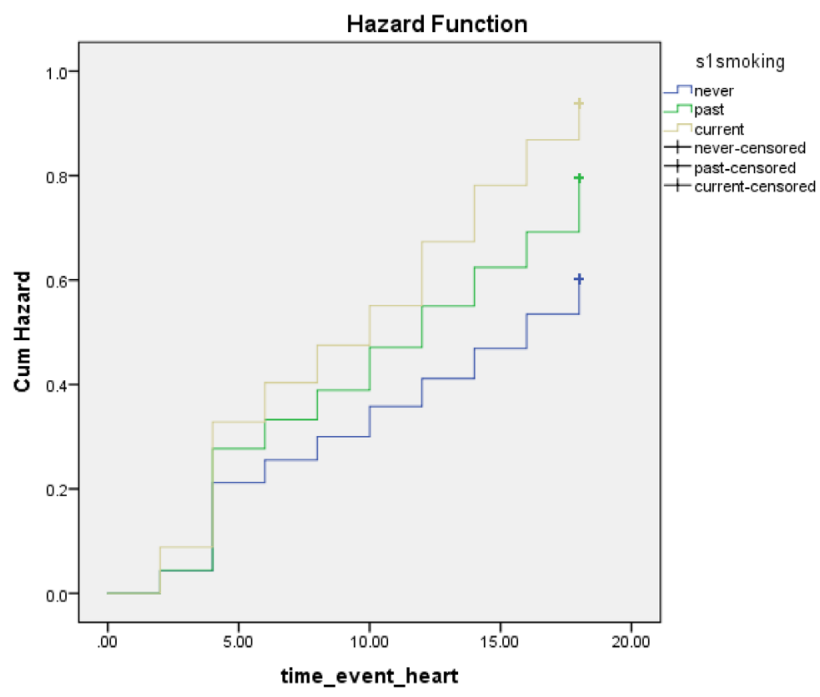

Never Smoker $=$ Blue, Past Smoker $=$ Green, Current Smoker $=$ Yellow

level of depressive symptoms at baseline was associated with CAD over a 20-year period. Our results suggested that this link remained significant after controlling for demographic data, socioeconomic characteristics, BMI, and health behaviors (i.e. exercise and smoking). These findings were in line with those studies that have shown that individuals who experience negative emotions, such as depression, were at an increased risk of CAD $(12,25,26)$. In a cohort of 7,709 patients with confirmed CAD but without a diagnosis of heart failure or depression, a subsequent diagnosis of depression was associated with $50 \%$ increase in the risk of heart failure (14). The Nurses' Health Study analysis also suggested that antidepressant use tripled the risk of sudden cardiac death in healthy women (15). Similarly, the Women's Ischemia Syndrome Evaluation 
Figure 4. Kaplan-Meier Graph Showing Hazard of Developing Heart Disease Over 20 Years Based on Level of Depressive Symptoms at Baseline $(\mathrm{n}=8,375)$

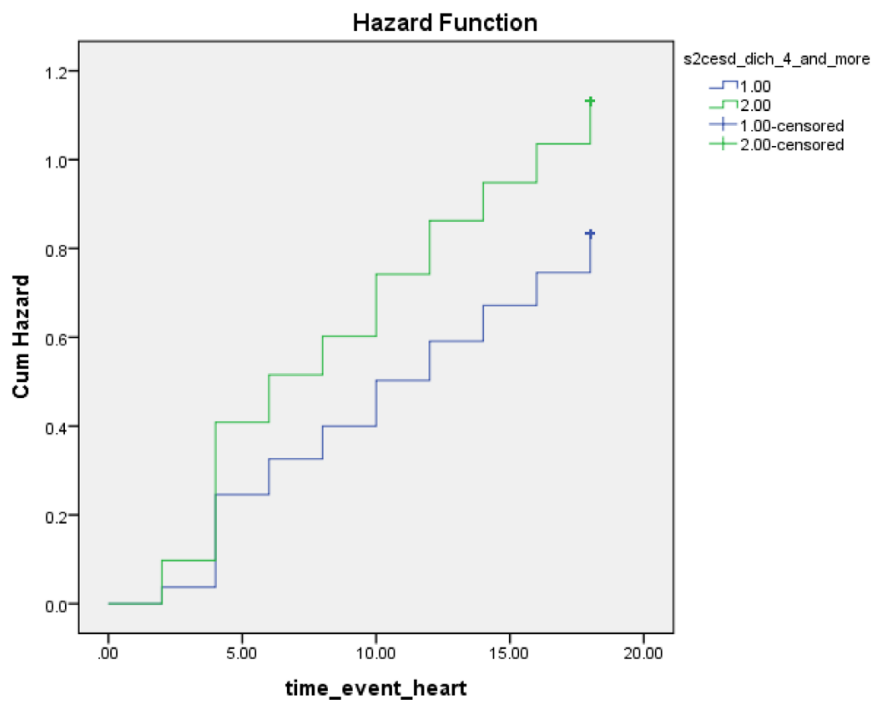

Low Depressive Symptoms = Blue, High Depressive Symptoms $=$ Green

\begin{tabular}{|c|c|c|c|c|c|}
\hline \multirow[b]{2}{*}{ High depressive symptoms } & \multirow{2}{*}{$\begin{array}{l}\text { HR } \\
1.439\end{array}$} & \multirow{2}{*}{$\begin{array}{l}\text { SE } \\
0.071\end{array}$} & \multirow{2}{*}{$\begin{array}{l}\text { Sig. } \\
<0.001\end{array}$} & \multicolumn{2}{|c|}{ 95\% Confidence Interval } \\
\hline & & & & 1.253 & 1.652 \\
\hline Female gender & 0.568 & 0.048 & $<0.001$ & 0.517 & 0.625 \\
\hline Age & 1.043 & 0.004 & $<0.001$ & 1.034 & 1.051 \\
\hline Blacks & 0.973 & 0.074 & 0.716 & 0.842 & 1.125 \\
\hline Hispanics & 0.668 & 0.100 & $<0.001$ & 0.549 & 0.812 \\
\hline Education & 0.969 & 0.008 & $<0.001$ & 0.954 & 0.984 \\
\hline \multicolumn{6}{|l|}{ Marital status } \\
\hline 1 & 2.182 & 0.356 & 0.028 & 1.086 & 4.383 \\
\hline 2 & 1.537 & 0.133 & 0.001 & 1.184 & 1.995 \\
\hline BMI & 1.022 & 0.004 & $<0.001$ & 1.014 & 1.030 \\
\hline Exercise & 1.029 & 0.007 & $<0.001$ & 1.015 & 1.043 \\
\hline \multicolumn{6}{|l|}{ Smoking } \\
\hline Past smoker & 1.184 & 0.052 & $<0.001$ & 1.070 & 1.310 \\
\hline Current smoker & 1.494 & 0.059 & $<0.001$ & 1.329 & 1.678 \\
\hline Drinking & 0.837 & 0.047 & $<0.001$ & 0.764 & 0.918 \\
\hline
\end{tabular}

revealed doubling of the risk of cardiovascular disease and death in the women taking antidepressants who had been referred for coronary angiography (13).

Several review articles have also been conducted on this topic. In a narrative review redundant. Rozanski et al. explained paths from psychosocial factors to CAD (25). Hemingway and Marmot also conducted a systematic review and showed that all the 11 eligible studies reported that psychological distress significantly increased the adjusted relative risk of development of coronary disease (9). Prospective studies have consistently suggested that negative affect predicts CAD $(25,27,28)$. Depression is associated with CAD morbidity and mortality, even after controlling for the traditional CAD risk factors, such as serum cholesterol, blood pressure, and smoking $(9,26,29)$. Although multiple behavioral pathways have been proposed to explain how depression is linked to development and progression of $\operatorname{CAD}(13,30)$, our study suggested that the link between depression and CAD may be independent of smoking, exercise, and BMI.

The findings of the current study may have important clinical or public health implications for primary prevention of heart disease in the community. Depression is the most common psychiatric disorder in the general population (31). About $20 \%$ of older adults in the US (aged $\geq 65$ years) have CAD $(32,33)$. Future studies should determine whether promotion of mental health in depressed individuals reduces morbidity and mortality associated with coronary heart disease in the populations. We argue that individuals with high depressive 
symptoms or depression who have not already developed heart disease should receive screening and evaluation for development of heart disease more closely compared to their non-depressed counterparts. Patients with depression (or high symptoms of depression) may benefit from targeted interventions with educational components on the risk of heart disease. To conclude, high level of depressive symptoms at baseline predicted new onset of heart disease over 20 years among the individuals older than 50 years in the United States.

\section{Acknowledgements}

For this analysis, the public data set was downloaded from Inter-university Consortium for Political and Social Research (ICPSR), Institute for Social Research at the University of Michigan. Health and Retirement Study was funded by the National Institute on Aging (NIA) (U01 AG09740).

\section{Authors' Contribution}

Maryam Moghani Lankarani and Shervin Assari designed the study and analyzed, drafted, and revised the manuscript.

\section{Financial disclosure}

There is no financial disclosure.

\section{Funding/Support}

Publication of this manuscript was possible with the postdoctoral fellowship awarded by Department of Psychiatry and the Center for Research on Ethnicity, Culture, and Health (CRECH), University of Michigan, to Shervin Assari. Shervin Assari also receives financial support by the Heinz C. Prechter Bipolar Research Fund and National Institutes of Health (NIH, grants HD084526-01 and DK089503).

\section{References}

1. Frasure-Smith N, Lesperance F. Recent evidence linking coronary heart disease and depression. Can J Psychiatry. 2006;51(12):730-7.

2. Barth J, Schumacher M, Herrmann-Lingen C. Depression as a risk factor for mortality in patients with coronary heart disease: a metaanalysis. Psychosom Med. 2004;66(6):802-13.

3. Nicholson A, Kuper H, Hemingway H. Depression as an aetiologic and prognostic factor in coronary heart disease: a meta-analysis of 6362 events among 146538 participants in 54 observational studies. Eur Heart J. 2006;27(23):2763-74.

4. Rugulies R. Depression as a predictor for coronary heart disease. a review and meta-analysis. Am J Prev Med. 2002;23(1):51-61.

5. Rutledge T, Reis VA, Linke SE, Greenberg BH, Mills PJ. Depression in heart failure a meta-analytic review of prevalence, intervention effects, and associations with clinical outcomes. J Am Coll Cardiol. 2006;48(8):1527-37.

6. van Melle JP, de Jonge P, Spijkerman TA, Tijssen JG, Ormel J, van Veldhuisen DJ, et al. Prognostic association of depression following myocardial infarction with mortality and cardiovascular events: a meta-analysis. Psychosom Med. 2004;66(6):814-22.

7. Wulsin LR, Singal BM. Do depressive symptoms increase the risk for the onset of coronary disease? A systematic quantitative review. Psychosom Med. 2003;65(2):201-10.

8. Glassman AH, Shapiro PA. Depression and the course of coronary artery disease. Am J Psychiatry. 1998;155(1):4-11.

9. Hemingway $\mathrm{H}$, Marmot $\mathrm{M}$. Evidence based cardiology: psychosocial factors in the aetiology and prognosis of coronary heart disease. Systematic review of prospective cohort studies. $B M J$. 1999;318(7196):1460-7.

10. Musselman DL, Evans DL, Nemeroff CB. The relationship of depression to cardiovascular disease: epidemiology, biology, and treatment. Arch Gen Psychiatry. 1998;55(7):580-92.
11. Rozanski A, Blumenthal JA, Kaplan J. Impact of psychological factors on the pathogenesis of cardiovascular disease and implications for therapy. Circulation. 1999;99(16):2192-217.

12. Suls J, Bunde J. Anger, anxiety, and depression as risk factors for cardiovascular disease: the problems and implications of overlapping affective dispositions. Psychol Bull. 2005;131(2):260-300.

13. Krantz DS, Whittaker KS, Francis JL, Rutledge T, Johnson BD, Barrow G, et al. Psychotropic medication use and risk of adverse cardiovascular events in women with suspected coronary artery disease: outcomes from the Women's Ischemia Syndrome Evaluation (WISE) study. Heart. 2009;95(23):1901-6.

14. May HT, Horne BD, Carlquist JF, Sheng X, Joy E, Catinella AP. Depression after coronary artery disease is associated with heart failure. J Am Coll Cardiol. 2009;53(16):1440-7.

15. Whang W, Kubzansky LD, Kawachi I, Rexrode KM, Kroenke CH, Glynn RJ, et al. Depression and risk of sudden cardiac death and coronary heart disease in women: results from the Nurses' Health Study. J Am Coll Cardiol. 2009;53(11):950-8.

16. Smoller JW, Allison M, Cochrane BB, Curb JD, Perlis RH, Robinson $\mathrm{JG}$, et al. Antidepressant use and risk of incident cardiovascular morbidity and mortality among postmenopausal women in the Women's Health Initiative study. Arch Intern Med. 2009;169(22):2128-39.

17. Barrick CB. Sad, glad, or mad hearts? Epidemiological evidence for a causal relationship between mood disorders and coronary artery disease. J Affect Disord. 1999;53(2):193-201.

18. Appels A. Depression and coronary heart disease: observations and questions. J Psychosom Res. 1997;43(5):443-52.

19. Hauser RM, Willis RJ. Survey design and methodology in the Health and Retirement Study and the Wisconsin Longitudinal Study. Population and Development Review. 2004:209-35.

20. Heeringa SG, Connor JH. Technical description of the health and retirement survey sample design. Ann Arbor: University of Michigan. 1995.

21. Radloff LS. The CES-D scale a self-report depression scale for research in the general population. Applied psychological measurement. 1977;1(3):385-401.

22. Yang FM, Jones RN. Measurement differences in depression: chronic health-related and sociodemographic effects in older Americans. Psychosom Med. 2008;70(9):993-1004.

23. Steffick D, Wallace R, Herzog A. Documentation of affective functioning measures in the Health and Retirement Study. 2000. Survey Research Center, University of Michigan Retrieved January. 2008;8.

24. Ayotte BJ, Yang FM, Jones RN. Physical health and depression: a dyadic study of chronic health conditions and depressive symptomatology in older adult couples. J Gerontol B Psychol Sci Soc Sci. 2010;65(4):438-48.

25. Chang PP, Ford DE, Meoni LA, Wang NY, Klag MJ. Anger in young men and subsequent premature cardiovascular disease: the precursors study. Arch Intern Med. 2002;162(8):901-6.

26. Kubzansky LD, Cole SR, Kawachi I, Vokonas P, Sparrow D. Shared and unique contributions of anger, anxiety, and depression to coronary heart disease: a prospective study in the normative aging study. Ann Behav Med. 2006;31(1):21-9.

27. Booth-Kewley S, Friedman HS. Psychological predictors of heart disease: a quantitative review. Psychol Bull. 1987;101(3):343-62.

28. Matthews KA. Coronary heart disease and type A behaviors: update on and alternative to the Booth-Kewley and Friedman (1987) quantitative review. Psychological bulletin. 1988;104(3):373.

29. Gallo LC, Matthews KA. Understanding the association between socioeconomic status and physical health: do negative emotions play a role? Psychol Bull. 2003;129(1):10-51.

30. Grippo AJ, Johnson AK. Biological mechanisms in the relationship between depression and heart disease. Neurosci Biobehav Rev. 2002;26(8):941-62.

31. Kessler RC, Berglund P, Demler O, Jin R, Koretz D, Merikangas $\mathrm{KR}$, et al. The epidemiology of major depressive disorder: results from the National Comorbidity Survey Replication (NCS-R). JAMA. 2003;289(23):3095-105.

32. CDC [cited October 7];Behavioral Risk Factor Surveillance System. 2011 Available from: http://www.cdc.gov/brfss.

33. Roger VL, Go AS, Lloyd-Jones DM, Adams RJ, Berry JD, Brown TM, et al. Heart disease and stroke statistics--2011 update: a report from the American Heart Association. Circulation. 2011;123(4):e18-e209. 